\title{
Stimulus and site specific induction of hiccups in the oesophagus of normal subjects
}

\author{
R Fass, L Higa, A Kodner, E A Mayer
}

\begin{abstract}
Background-Hiccups that are induced by a large meal have been suggested to result from gastric overdistension. The role of the oesophagus in precipitating hiccups has never been defined.

Aims-To determine the involvement of oesophageal mechanoreceptors in the hiccup reflex.

Methods-Ten normal healthy subjects were prospectively evaluated at a university affiliated hospital. Controlled inflation of a polyethylene bag in the proximal and distal oesophagus was carried out using slow ramp and rapid phasic distensions, by an electronic distension device.

Results-Hiccups were induced in four subjects only during rapid phasic distensions and only in the proximal oesophagus. The mean (SEM) minimal volume threshold for the hiccup reflex was 32.5 (4.8) $\mathrm{ml}$, which was above the perception threshold. Hiccups appeared during inflation and resolved after deflation.

Conclusions-Sudden rapid stretch of the mechanoreceptors in the proximal oesophagus can trigger the hiccup reflex in normal subjects. Only rapid distensions above a determined volume threshold will predictably induce hiccups in a given subject. This mechanism may play a role in the physiological induction of hiccups. (Gut 1997; 41: 590-593)
\end{abstract}

Digestive Disease Research

Center/Neuroenteric Biology Group, Department of Medicine, UCLA and West LA VA Medical Center, Los Angeles, California, USA

L Higa

A Kodner

E A Mayer

Section of Gastroenterology (111G-1), Tucson VA

Medical Center, Tucson, USA

$\mathrm{R}$ Fass

Correspondence to: Dr R Fass, Section of Gastroenterology (111G-1), Tucson VA Medical Center, 3601 S. 6 th Ave., Tucson, AZ 85723, USA.

Accepted for publication 12 May 1997 nection is the spinal cord between segments C3 and C5, possibly controlled by supraspinal pathways. ${ }^{12}$ Excessive food and alcohol consumption, ingestion of carbonated beverages, and aerophagia can trigger the hiccup reflex. ${ }^{1{ }^{2} 7}$ Several changes have been observed in the function of the oesophagus during hiccups: a complete disappearance of oesophageal body peristalsis, decreased lower oesophageal sphincter pressure, and a fluctuation in the oesophageal baseline, synchronous with hiccups activity. ${ }^{89}$ These functional changes are transient and disappear after hiccup cessation.

Hiccups may be induced by rapid swallowing of a large bolus of food. Although gastric overdistension has been suggested as a possible aetiological mechanism, the role of the oesophagus in triggering the hiccup reflex remains to be elucidated. ${ }^{12}$ The aim of our study was to characterise the stimulus and the oesophageal anatomical location which may be responsible for triggering the hiccup reflex. A better understanding of the physiological role of the oesophagus in inducing hiccups may improve the management of self limited or intractable hiccups of oesophageal origin.

\section{Methods}

Ten normal, healthy subjects (eight males and two females; age range $19-54$ years, mean age 35.1 (12.2) years) were recruited. All subjects were interviewed to exclude a history of chronic hiccups, diaphragmatic irritation, central nervous system abnormality, gastrooesophageal reflux disease, upper gastrointestinal tract surgery, active peptic ulcer disease, autonomic or peripheral neuropathy, myopathy, diabetes mellitus, and any other disorder or medication use that may induce hiccups or gastro-oesophageal reflux, or affect sensation. An essential inclusion criterion was normal 24 hour oesophageal $\mathrm{pH}$ monitoring to exclude asymptomatic gastro-oesophageal reflux disease. After an overnight fast, a $\mathrm{pH}$ probe was inserted via the nose into the stomach. The $\mathrm{pH}$ probe was connected to a digital portable recorder (Synectics Medical, Digitrapper Mark II). Using the pull through technique, the probe was placed $5 \mathrm{~cm}$ above the point of sudden rise in $\mathrm{pH}$. A reference electrode was attached to the upper chest. The subjects were instructed to keep a diary, recording meal time and position changes. Subjects were encouraged to pursue their everyday activities and usual diet. At the beginning and the end of the study the electrode and the system were calibrated in standard solutions of $\mathrm{pH} 1$ and $\mathrm{pH} 7$. A study was considered abnormal when the percentage total time of $\mathrm{pH}<4$ was greater than $4.2 \%$.

The oesophageal distension protocol was carried out after an overnight fast, using a 
computer driven volume displacement device (electronic barostat, Synectics Visceral Stimulator; Synectics, Stockholm, Sweden) which was used to inflate a bag either continuously at a constant volume rate of $80 \mathrm{ml} / \mathrm{min}$ (ramp distension) or rapidly ( $870 \mathrm{ml} / \mathrm{min})$ to constant volume plateaus (phasic distension). The distension device, which records pressures and volumes simultaneously (sampling rate of one per second) was connected to a subject operated marker device that logs the sensation of initial balloon inflation, discomfort, and pain onto the data file as bag inflation occurs. When a subject triggered the marker for pain during phasic or ramp distension, the device instantaneously deflated. There was a fixed pressure limit that also triggered bag deflation for pressures greater than $80 \mathrm{~mm} \mathrm{Hg}$.

A polyethylene bag was attached to a plastic probe (diameter 10F) and tied at both proximal and distal ends (MAK-LA, Los Angeles, California). The distance between the two attachment sites was $5.5 \mathrm{~cm}$. Distension to a maximal volume of $150 \mathrm{ml}$ and circumference of $27 \mathrm{~cm}$ resulted in a spherical bag shape. However, partial inflations resulted in a cylindrical shape in vivo. The bag was mounted over three ports used for inflation and intrabag pressure and volume measurements. The bag was inflated repeatedly before initial use and after completion of each experiment to ensure that there were no leaks.

Subjects were placed in the prone position on a padded table. The bag was inserted via the mouth and positioned initially in the distal ( $5 \mathrm{~cm}$ above lower oesophageal sphincter) and subsequently in the proximal $(15 \mathrm{~cm}$ above lower oesophageal sphincter) oesophagus. The tube was secured each time in its proper position with tape. The location of the lower oesophageal sphincter was predetermined by oesophageal manometry. Patients were not informed about the nature of the distension protocols (ramp distension versus random phasic distension). During each protocol, subjects had full control and were able to

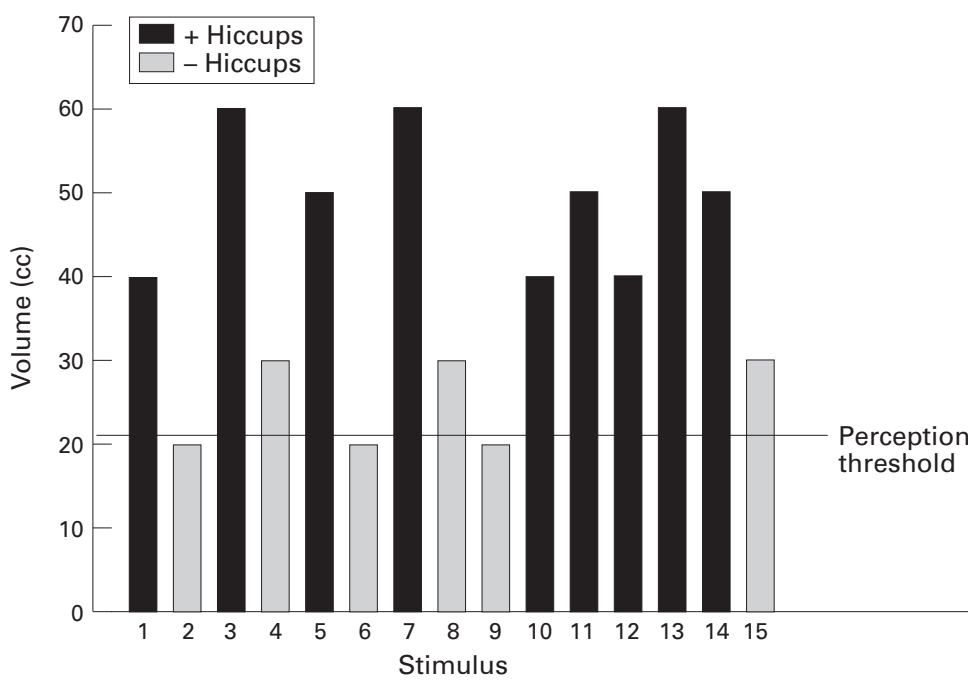

Figure 1: Stimulation of hiccups by rapid, phasic distensions in the proximal oesophagus of a single subject. The black bars represent phasic distensions that induced hiccups. The hiccup volume threshold is above the perception threshold. terminate the test at any time of significant discomfort.

Initially, a slow ramp distension at a rate of $80 \mathrm{ml}$ per minute was delivered until the subject pressed the pain button. The hiccups volume threshold, if precipitated, and the perception thresholds for initial sensation and discomfort were recorded. Perception threshold for initial sensation was defined as the lowest volume that induced oesophageal sensation. The slow ramp distension was repeated three times to determine reproducibility. Subsequently, a predetermined protocol of randomly delivered phasic distensions, at a rate of $870 \mathrm{ml}$ per minute, was carried out in the proximal and distal oesophagus. Each stimulus lasted $30 \mathrm{sec}-$ onds, followed by a 30 second resting interval. Five different volumes (20, 30, 40, 50, and $60 \mathrm{ml}$ ) were randomly delivered, such that each stimulus was given three times. During each phasic stimulus, subjects were requested to report their perception. Repetition of each protocol was carried out to ensure reproducibility of the hiccups, if precipitated. After completion of each protocol in the distal oesophagus, the balloon was withdrawn and placed $15 \mathrm{~cm}$ above the lower oesophageal sphincter (proximal oesophagus). Similar protocols were performed in the proximal oesophagus.

Data analysis was performed using Fisher's exact test and Student's $t$ test. Results are presented as mean (SEM). All subjects gave informed consent and the study was approved by the Human Subject Committee of the West Los Angeles Veteran Administration Medical Center.

\section{Results}

Ten healthy subjects completed the study. Hiccups were not induced during slow ramp distension in the distal or the proximal oesophagus in any of the subjects. During rapid phasic distension, hiccups were induced in the proximal oesophagus of four subjects $(40 \%)$. In contrast, hiccups were not induced by rapid phasic distension in the distal oesophagus of any subject, including those who developed hiccups in the proximal oesophagus.

There was no significant age difference between subjects who developed hiccups and those who did not (38.0 (9.0) and 34.0 (5.0) respectively). All four subjects who developed hiccups were males. The mean perception thresholds for initial sensation in the proximal and distal oesophagus were similar for all subjects (15.2 (1.7) and 19.8 (2.7) $\mathrm{ml}$ respectively). However, the mean threshold for discomfort was significantly higher in the distal than the proximal oesophagus for all subjects (31.3 (2.5) and 23.7 (2.1) $\mathrm{ml}$ respectively). During rapid phasic distension, the mean threshold for hiccups was 32.5 (4.8) $\mathrm{ml}$, which was significantly above the threshold for initial perception $(p<0.05)$ (fig 1$)$. The mean intrabag pressure thresholds for hiccups did not differ from the intrabag pressures generated by similar volumes in subjects that did not develop hiccups (64.4 (7.2) and 62.1 (7.0) $\mathrm{mm} \mathrm{Hg}$, respectively). Only minimal contractile response to the different stimuli was recorded in 
the distal oesophagus of one subject out of the four that developed hiccups. In the proximal oesophagus, contractile response was recorded in $2 / 4$ subjects, none of which had any detectable response in the distal oesophagus. Conclusions about contractile response to the different stimuli are thus hampered by the very small number of subjects. There was no significant difference in the mean perception thresholds for initial sensation and discomfort in the proximal oesophagus of the subjects who developed hiccups when compared with those who did not (18.2 (2.6) and 23.8 (2.9) versus $12.9(2.2)$ and $24.3(2.8) \mathrm{ml}$ ).

Phasic distensions greater than $40 \mathrm{ml}$ induced hiccups in all four subjects in whom hiccups occurred. The hiccups were induced during the rising phase of inflation of the bag and immediately resolved after deflation. None of the participants who developed hiccups continued to experience them after completion of the protocols. Reproducibility of hiccups was achieved in all subjects who developed them initially, at the same location and after the same phasic stimulus. None of the subjects not experiencing hiccups during the initial set of slow or rapid distensions developed hiccups during any of the repeated protocols.

\section{Discussion}

The role of the oesophagus in precipitating hiccups has never been defined. The usual, brief, and transient hiccups that are induced by a large meal or carbonated beverages, have been suggested to result from overdistension of the stomach. ${ }^{12}$ However, careful history of patients with hiccups, suggests that meal induced hiccups may be related to the size and the rapidity of a swallowed bolus rather than the overall amount of food that has been ingested. This study is the first to demonstrate a clear role for the proximal oesophagus in precipitating hiccups. Furthermore, the ability to induce hiccups by rapid phasic distension limited to the proximal oesophagus suggests that a sudden, rapid stimulation of oesophageal mechanoreceptors by either a large bolus of food or large amount of air, as in carbonated beverages, induces hiccups. Since we did not perform gastric distension in these subjects, we cannot rule out that hiccups in a given individual can be triggered by both oesophageal and gastric distension. However, in separate studies using the same protocols for gastric distension stimulation in healthy volunteers, we never observed the induction of hiccups. ${ }^{11}$ In addition, it appears from our study that the mechanoreceptors responsible for triggering hiccups respond to a defined volume threshold which is above the perception threshold.

Even though compliance and perception thresholds are similar in both distal and proximal oesophagus in normal subjects, rapid balloon distension in the distal oesophagus did not induce hiccups. ${ }^{10}$ A possible explanation is selective innervation of the proximal oesophagus by vagal or sympathetic afferents which participate in the hiccup reflex arc. The fact that hiccups were induced in only four of the volunteers may be related to a higher hiccup threshold in the non-responsive subjects.

Several reports have associated hiccups with gastro-oesophageal reflux disease (GORD). ${ }^{12-16}$ Reproducibility of hiccups was demonstrated by stimulation of oesophageal chemoreceptors by acid perfusion in a patient with GORD who suffered from a persistent form of hiccups. ${ }^{13}$ However, the association remains controversial as some authors hypothesised that hiccups themselves predispose to acid reflux, while others could not demonstrate improvement in hiccups with medical or surgical antireflux treatment. ${ }^{12} 14$ In the current study, we have eliminated both symptomatic and asymptomatic GORD as a cause for the observed findings.

Achalasia, stricture, and malignant tumours confined to the oesophagus have been associated with intractable hiccups in sporadic case reports. ${ }^{17-19}$ Dilatation of the oesophagus has been implicated as the underlying mechanism. ${ }^{19}$ In these reports, patients developed hiccups after meals, suggesting an increased predisposition to sudden stretching of the proximal oesophagus due to oesophageal obstruction or lack of normal oesophageal peristalsis.

The physiological role of hiccups has never been determined. However, our study may suggest a protective mechanism, whereby hiccups possibly prevent very large boluses of food from entering the gastrointestinal tract. In summary, this is the first study to demonstrate the involvement of oesophageal mechanoreceptors in triggering hiccups. By understanding this physiological mechanism in patients with self limited or persistent hiccups of presumed oesophageal origin, one could suggest initial therapeutic measures in the form of chewing well and swallowing slowly small boluses of food. In cases of persistent symptoms, identification of possible underlying pathology remains mandatory.

This study was supported by a research grant from Astra/Merck and by funds from the Veterans Administration. Presented in part at the annual meeting of The American Gastroenterological Association, San Francisco, California, May 1996.

1 Loft LM, Ward RF. Hiccups. Arch Otolaryngol Head Neck Surg 1992; 118: 11 15-9.

2 Kolodzik PW, Eilers MA: Hiccups (singultus): review and approach to management. Ann Emerg Med 1991; 20: 56573.

3 Odeh M, Bassan H, Oliven A. Termination of intractable hiccups with digital rectal massage. $\mathcal{F}$ Int Med 1990; 227: 145-6.

4 Ramirez F, Grahm DY. Treatment of intractable hiccup with baclofen: results of a double-blind randomized, controlled, cross-over study. Am f Gastroenterol 1992; 87: 1789-91.

5 Sato S, Asakura N, Endo T, Naito H. Cervical epidural block can relieve postoperative intractable hiccups. $A n$ block can relieve postoperative
esthesiology 1993; 78: 1184-6.

esthesiology 1993; 78: 1184-6.
6 Johnson DL. Intractable hiccups: treatment by microvascular decompression of the vagus nerve. $\mathcal{F}$ Neurosurg 1993; 78: $813-6$.

7 Lewis JH. Hiccups: causes and cures. $\mathcal{F}$ Clin Gastroenterol 1985; 7: 539-52.

8 Grahm DY. Oesophageal motor abnormality during hiccup. Gastroenterology 1986; 90: 2039-44.

9 Triadafilopoulos G. Hiccups and oesophageal dysfunction. Am 7 Gastroenterol 1989; 84: 164-9.

10 Fass R, Munakata J, Naliboff B, Kodner A, Sytnik B, Perez $M$, Mayer EA. Characterization of oesophageal afferent function in normal subjects. Am $\mathcal{F}$ Gastroenterol 1995; 90: 1574 .

11 Mertz H, Kodner A, Hirsh T, Sytnik B, Mayer EA. Gastric balloon distension as a diagnostic test for non-ulcer dyspepsia [abstract]. Gastroenterology 1994; 106: A539.

12 Shay SS, Myers RL, Johnson LF. Hiccups associated with reflux esophagitis. Gastroenterology 1984; 87: 204-7. 
13 Gluck M, Pop II CE. Chronic hiccups and gastroesophageal reflux disease: the acid perfusion test as a provocative maneuver. Ann Intern Med 1986; 105: 219-20.

14 Fisher MJ, Mittal RK. Hiccups and gastrooesophageal reflux: cause and effect? Dig Dis Sci 1989; 34: 1277-80.

15 Marshall JB, Landreneau RJ, Beyer KL. Hiccups: oesophageal manometric features and relationship to gastroesophageal reflux. Am F Gastroenterol 1990; 85: 1172-5.
16 Zaninotto G, Costantini M, Ancona E. Hiccups and related esophageal motor disorders. Hepatogastroenterology 1991;

17 McLoughlin JC, McGuigan J. Hiccups and oesophageal malignancy. Ulster Med f 1991; 60: 246-7.

18 Kaufmann HJ. Hiccups: an occasional sign of esophageal obstruction. Gastroenterology 1982; 82: 1443-5.

19 Seeman H, Traube M. Hiccups and achalasia. Ann Intern Med 1991; 115: 711-2. 